\title{
Economic evaluation of sunitinib versus pazopanib and best supportive care for the treatment of metastatic renal cell carcinoma in Chile: cost- effectiveness analysis and a mixed treatment comparison
}

C Vargas, C Balmaceda, F Rodríguez, R Rojas, A Giglio \& MA Espinoza

To cite this article: C Vargas, C Balmaceda, F Rodríguez, R Rojas, A Giglio \& MA Espinoza (2019): Economic evaluation of sunitinib versus pazopanib and best supportive care for the treatment of metastatic renal cell carcinoma in Chile: cost-effectiveness analysis and a mixed treatment comparison, Expert Review of Pharmacoeconomics \& Outcomes Research, DOI: 10.1080/14737167.2019.1580572

To link to this article: https://doi.org/10.1080/14737167.2019.1580572

Accepted author version posted online: 13

Feb 2019.

Submit your article to this journal ¿

View Crossmark data $\nearrow$ 
Publisher: Taylor \& Francis

Journal: Expert Review of Pharmacoeconomics \& Outcomes Research

DOI: $10.1080 / 14737167.2019 .1580572$

Original Research

Economic evaluation of sunitinib versus pazopanib and best

supportive care for the treatment of metastatic renal cell

carcinoma in Chile: cost-effectiveness analysis and a mixed

treatment comparison

Vargas $\mathrm{C}^{1,2}$, Balmaceda $\mathrm{C}^{1}$, Rodríguez $\mathrm{F}^{3}$, Rojas $\mathrm{R}^{1}$, Giglio $\mathrm{A}^{4}$, Espinoza $\mathrm{MA}^{1,5}$

${ }^{1}$ Health Technology Assessment Unit, Center of Clinical Research, Faculty of Medicine, Pontificia Universidad Católica de Chile

${ }^{2}$ Centre of Health Economics Research and Evaluation (CHERE), University of Technology Sydney, Sydney.

${ }^{3}$ Faculty of Medicine, Universidad San Sebastián

${ }^{4}$ Programa de Medicina Interna, Complejo Asistencial Sótero del Río

${ }^{5}$ Department of Public Health, Faculty of Medicine, Pontificia Universidad Católica de Chile

Corresponding author:

Manuel Espinoza

Department of Public Health

Health Technology Assessment Unit, Center of Clinical Research, Faculty of Medicine, Pontificia Universidad Católica de Chile

Diagonal Paraguay 362, 2 $2^{\text {nd }}$ Floor, Santiago, Chile.

Tel: +562 2354-6807

Email:maespinoza@med.puc.cl 


\section{Abstract}

BACKGROUD: Sunitinib and Pazopanib are two metastatic renal cell carcinoma (MRCC) treatment alternatives, however the health system in Chile does not consider coverage for any. The cost-effectiveness versus relevant comparator was assessed to support evidencebased decision making.

METHODS: A four health states Markov model was built: first, second line treatments, BSC and death. Benefits were measured in QALYs and efficacy estimates were obtained from an indirect treatment comparison. A 10-year time horizon and a 3\% undifferentiated discount rate were considered. Deterministic and probabilistic sensitivity analyses were performed.

RESULTS: The costs of treating MRCC with Sunitinib were higher than Pazopanib and BSC. When comparing Sunitinib versus Pazopanib, the incremental benefit is small favoring Sunitinib (0.03 QALYs). The base case scenario shows an average ICER of PA versus BSC of US\$62,327.11/QALY and of US\$85,885/QALY for Sunitinib versus Pazopanib. The ICER was most sensitive to the OS relative to $\mathrm{BSC}$, where evidence was associated to important bias. CONCLUSIONS: Sunitinib or Pazopanib can be considered cost-effective if a 3 GDP per-capita threshold is assumed. The decision between SU or PA is highly sensitive to the price of the drugs, rather than the outcomes. Therefore, the decision might be made based on costminimization exercise.

Keywords Health Technology Assessment, Cost-effectiveness, Renal cell carcinoma, Sunitinib, Receptor tyrosine kinase inhibitors, Pazopanib. 


\section{$1 \quad$ Introduction}

Renal cell carcinoma (RCC) is the most common type of kidney cancer accounting for approximately $90 \%$ of the total renal cancers. It represents $2-3 \%$ of all cancers with an incidence of 4.4 per 100,000 habitants, which is even greater in developed countries[1-3]. It is associated with a mortality rate of 3.1 per 100,000 habitants, which also increases exponentially in people over 50 years[3]. The most relevant associated risk factors are tobacco consumption, obesity and hypertension[4-6]. In Chile, RCC has an incidence of six per 100,000 habitants and is more commonly diagnosed in men at an age ranging between 60 and 70 years $[3,7]$.

Patients with stage I RCC have a 5-year survival rate between 80 to 95\%, stage II 80\%, stage III 60\% and stage IV (patients with metastasis - MRCC) less than $10 \%$ with a median overall survival of 10 to 15 months[8]. Furthermore, nearly $30 \%$ of patients have metastases at diagnosis, and $40 \%$ of patients with localized tumor will develop metastases at some point of their disease, mainly lung, bone, liver or brain[9].

Prior to the approval of targeted therapies, interferon alpha (IFNa) was used as first-line treatment for MRCC. However, it showed a limited effectiveness (10-15\%) and was associated with high rate of discontinuation due to adverse events[10]. High-dose Interleukin-2 (IL-2)

showed an overall response rate of $15 \%$ and $1 \%$ probability of death due to toxicity. Hence, its use was limited to young patients with good performance status and who had limited metastasis[8,11,12]. Vascular endothelial growth factor (VEGF) inhibitors such as Bevacizumab (BE) associated with IFN $\alpha$, Sorafenib (SO), Sunitinib (SU) and Pazopanib (PA) have shown improved effectiveness compared with former treatments[11]. Currently, based 
on available experience, SU and PA are the most accepted treatments and considered the standard of care in most countries.

In Chile, SU and PA were approved by the regulatory agency as first-line treatments for MRCC in 2006 and 2013 respectively. The evidence for SU supporting this approval, comes from two main Phase III randomized controlled trials (RCT). The first assessed the efficacy, safety and overall survival (OS) of SU compared to IFN $\alpha$ in patients with MRCC who had no prior treatment[13]. The results showed a significantly higher PFS in the group of SU with a HR of $0.42(95 \%$ CI $0.32-0.54 ; \mathrm{p}<0.001)$ and objective response rate of $31 \%$ for SU and $6 \%$ for IFN $\alpha$. The second study showed the updated OS results with a non-statistically significant HR of 0.821 (95\% CI $0.673-1.001 ; p=0.051)[14]$. On the other hand, PA was assessed in two studies that compared it with placebo (PL) in patients with MRCC with intermediate or favorable prognosis[15,16]. The reported time to progression reached 9.2 and 4.2 months respectively (HR $0.46,95 \% \mathrm{CI} 0.34-0.62 ; \mathrm{p}<0.0001$ ). The overall response rate was $30 \%$ for PA and 3\% for PL, with a median response duration of 58.7 weeks. Finally, the efficacy PA versus SU was directly compared in a randomized non-inferiority clinical trial, showing noninferiority of PA compared to SU either for PFS (HR 1.05 95\% CI 0.9 - 1.22) or OS (HR 0.91 95\% CI $0.76-1.08 ; p=0.28$ [17]. This study also claimed that PA had a better safety profile and health-related quality-of-life, but a higher proportion of treatment discontinuation due to adverse events (24\% vs $20 \%)[17]$.

Currently, the public health system in Chile does not reimburse any targeted therapy to patients with MRCC who are currently only managed with palliative care. Moreover, there are no renal cancer clinical guidelines or an established treatment protocol available to guide the management of these patients. However, most international clinical guidelines and Health Technology Assessment (HTA) reports have recommended the use of BE/IFN $\alpha$ or PA as firstline treatment in patients with MRCC with favorable or moderate prognosis and SU as first- 
line treatment for any histological type of MRCC $[1,18,19]$. In this context, the question whether to fund SU or PA or both becomes extremely relevant. In order to inform this decision, we performed a cost-effectiveness analysis of SU versus PA and BSC (the current practice in Chile) as first-line treatment of MRCC from the perspective of the Chilean public health care system.

\section{$2 \quad$ Patients and Methods}

\subsection{Decision Model}

A time-dependent Markov model was used consisting of four health states: (1) patients receiving first-line treatment, (2) patients receiving second-line treatment, (3) patients receiving best supportive care (BSC) and (4) specific-cause death (Figure 1). A hypothetical cohort of MRCC patients was simulated and simultaneously treated with either SU, PA or BSC. It was assumed that BSC was equivalent to PL in terms of OS and PFS. The model assumed that patients received first-line treatment until disease progression or death. Patients who progressed after first-line treatment received second-line treatment, BSC or die. Similarly, patients receiving second-line treatment remained in that state until disease progression (BSC) or death. Patients that progress remain in this state until death. The model was

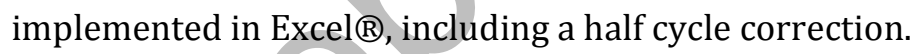

\section{Figure 1 here}

\subsection{Study Perspective and discount rate.}

The study was conducted from the perspective of the Chilean public health care system and the costs and benefits were discounted at an annual rate of $3 \%$ as suggested in the Chilean economic evaluations guideline[20]. A deterministic sensitivity analysis was also performed considering $0 \%$ and $6 \%$ rates. 


\section{$2.3 \quad$ Treatment Effect}

Because there are no "head-to-head" studies comparing targeted therapy (SU or PA) versus the current practice in Chile, which is BSC, as first-line treatment for MRCC, we conducted a literature search that included all possible treatment alternatives to implement a mixed treatment comparison (MTC). This methodology allows the estimation of a treatment effect between comparators that have not been previously compared in head-to-head trials. Efficacy was reported based on the proportion of patients who did not progress (PFS_HR) or if progressed, did not die (OS_HR). The MTC was implemented in Winbugs $®$ version 1.4 .

A total of 12 head-to-head studies assessing seven possible MRCC treatments were identified: IFN $\alpha$, PA, Temsirolimus (TEM), BE/IFN $\alpha$, SO and PL (for the purpose of this study assumed equivalent to BSC). Two studies were excluded because the study population did not share the same baseline characteristics relative to the SU study, a critical aspect to conduct MTC. First, the study by Hudes et al that compared TEM with IFN $\alpha$ and the combination of both, considered a patient population with a more unfavorable risk profile (MSKCC $\geq 3$ )[21]. Second, the study that compared SO vs PL evaluated previously treated patients (IL-2/IFN $\alpha$ as first line of treatment)[22]. All the included studies were phase III RCTs. According to data availability, it was possible to construct the network of comparators for PFS and OS as shown in Figure 2-A and Figure 2-B respectively.

\section{Figure 2-A and 2-B here}

\subsection{Survival Analysis}

Regression coefficients obtained from individual patient data (IPD) were provided from the SU clinical trials which reported efficacy up to 36 weeks[13,14]. The long-term treatment effect was modelled by adjusting a parametric distribution to the observed Kaplan Meier assuming the proportional hazards assumption holds. The Weibull distribution produced the 
best goodness of fit among several tested parametric functions[23]. The probability of progressing from first-line treatment was obtained from the difference between the probability of non-progression, given by PFS, and the probability of death, given by OS.

\section{$2.5 \quad$ Costs}

Costs were measured in 2014 Chilean pesos adjusted by consumer price index and converted into US dollars using average 2014 exchange rate (1 USD $=654.07$ CLP)[24]. The main sources of information for the identification of resources were: (i) the Chilean cost study used to estimate the per patient premium for the national health benefit plan; (ii) the 2014 public payer (FONASA) tariffs available for a limited list of health services/resources; and (iii) the ESMO clinical practice guidelines for the management of RCC $[25,26]$. This information was supplemented by other international literature when required. The price of treatments were requested to the national procurement entity (CENABAST) who provided the average 2014 price for each medication.

\subsection{Utility values}

Health related quality of life (HRQoL) estimates were obtained from patient data collected from two SU clinical trials[17,27]. These studies measured HRQoL using the EQ-5D and valued using the British tariff. As the trial did not consider PA, it was assumed that the quality of life of these patients corresponded to the weighted average utility of patients receiving SU during each cycle of 6 weeks ( 4 weeks on treatment plus 2 weeks off treatment). In order to assess possible uncertainties around these point estimates, a review of the literature was conducted to obtain additional parameters which were later tested in the deterministic sensitivity analysis.

\subsection{Sensitivity Analysis}


We conducted a deterministic sensitivity analysis with variations of $\pm 30 \%$ relative to the point estimate in all relevant parameters. A probabilistic sensitivity analysis was also performed to characterize second order uncertainty.

\section{$2.8 \quad$ Baseline Scenario}

For the economic analysis, the base case was constructed based on the recommendations established in the economic evaluation guideline[20,25]. Based on clinical experts opinions, this study assumed that after progressing from first-line treatment, patients could only receive BSC. The main arguments supporting this assumption was that the net benefit associated to second line treatments was still unclear. In addition, the current relevant question in Chile refers to first line. A scenario analysis was conducted to assess the effect on the results of transiting through a second line health state. Survival curves were constructed using the regression parameters obtained from the study that compared SU versus IFN. For extrapolation beyond trial duration, a 10-year time-horizon was considered. Table 1 summarizes the baseline scenario assumptions.

Table 1. Base line scenario: model input parameters and assumptions

\begin{tabular}{|l|l|l|}
\hline Parameter & Base case & Assumption/ Comment \\
\hline Discount rate & $3 \%$ & $\begin{array}{l}\text { Recommended in the local } \\
\text { economic evaluation } \\
\text { guideline[20]. }\end{array}$ \\
\hline $2^{\text {nd }}$ line probability & $0 \%$ & Experts input. \\
\hline $\begin{array}{l}\text { Regression coefficients from } \\
\text { IPD to build survival curves }\end{array}$ & SU vs IFN & $\begin{array}{l}\text { Available data provided in the } \\
\text { model. }\end{array}$ \\
\hline Survival extrapolation & Weibull & $\begin{array}{l}\text { As per visual inspection and } \\
\text { expert's opinion. }\end{array}$ \\
\hline HR PA versus PL & $\begin{array}{l}0.43 \text { (95\% CI 0.215 - 1.388; } \\
\text { p=0.172) }\end{array}$ & $\begin{array}{l}\text { Adjusted by cross over } \\
\text { (RPSFT)[16] }\end{array}$ \\
\hline Time Horizon & 10 years & Experts input \\
\hline SU posology & $\begin{array}{l}4 \times 2 \text { (4 weeks on treatment } \\
\text { followed by 2 weeks off treatment) }\end{array}$ & Approved posology in Chile[29] \\
\hline PA posology & 800 mg daily until progression & Approved posology in Chile[30] \\
\hline
\end{tabular}




\begin{tabular}{|l|l|l|}
\hline Comparators & - PA & Experts input \\
& - BSC & \\
\hline
\end{tabular}

Abbreviations: BSC: best supportive care; HR: hazard ratio; IFN: interferon; IPD: individual patient data; mg; milligrams; N/A: not applicable; PA: pazopanib; PL: placebo; RPSFT: Rank Preserving Structural Failure Time; SU: sunitinib.

\subsection{Second line treatment scenario}

A scenario analysis was conducted assuming $50 \%$ of patients were suitable to receive treatment with Axitinib (AX). Based on published data, it was assumed that OS was equivalent to the survival curve of patients receiving IFN $\alpha$ as first-line treatment. This assumption was supported by the visual inspection of the curves of the study that compared AX versus SO and the study that compared SU versus IFN $\alpha$ as well as expert opinion[13,14,31].

\section{$3 \quad$ Results}

\subsection{Treatment effect - Mixed Treatment Comparison}

A detailed analysis of each study was conducted to identify the best point estimate to be included in the MTC. A relevant aspect to highlight regarding the study comparing SU vs IFN $\alpha$ was the fact that some patients who initially received IFN $\alpha$, changed to SU (cross-over)[14]. For this reason, an adjusted by cross-over (0.808; 95\% CI 0.661-0.987; $\mathrm{p}=0.036)$, intention to treat (ITT) (OS HR: $0.821 ; 95 \%$ CI $0.673-1.001 ; \mathrm{p}=0.051)$ and a post hoc analysis $(0.647$; 95\% CI: 0.483-0.870; $\mathrm{p}=0.003$ ) OS HR were reported. The latter estimate only included patients who did not receive SU after the study ended. Finally, the MTC was conducted maintaining a conservative approach by using the ITT HR as the OS treatment effect.

A critical aspect was also identified in the study that compared PA versus PL, where the ITT analysis for OS did not show significant differences (HR: 0.91; 95\% CI 0.7-1.16; p=0.224)[16]. This study considered treatment naïve and cytokine pre-treated patients. This was partly explained by the cross-over of $54 \%$ of placebo patients to PA after disease progression was established through an open (non-blind) study extension. An adjusted HR using the RPSFT 
(Rank preserving structural failure time) methodology was reported (OS HR: 0.432; 95\%CI 0.215-1.388; $\mathrm{p}=0.172$ ). This methodology was considered adequate based on recommendations that suggest to use this approach when there is a high proportion of crossover $(>50 \%)[32,33]$. Additionally, it was considered inappropriate to use the adjusted HR in the naive population (OS HR: $0.31 ; 95 \%$ CI $0.073-1.715$; $\mathrm{p}=$ not reported) due to the great uncertainty observed (limited number of patients analysed) and possible risk of treatment effect overestimation.

Finally, despite statistically significant differences were not found in the HR ratio reported in the non-inferiority study of PA vs SU, the trial met good standards for internal validity. Hence, the treatment effect estimate was considered a useful parameter from the perspective of an economic evaluation (OS HR 0.91; 95\% CI: 0.76 - 1.08: $p=0.28$ )[17]. The main difference between non-inferiority and superiority trials relates to the statistical power and confidence, which affects statistical inference. Therefore, because we propagated second order uncertainty in a Bayesian statistical framework and we focused on the a-posteriori expected outcomes, those concerns for statistical inference were considered no longer relevant for the cost-effectiveness results. Instead, we should focus our interpretative efforts on the results of the probabilistic sensitivity analysis.

The results of the MTC for PFS and OS are presented in Table 2. This table shows the results comparing each treatment versus the baseline IFN $\alpha$ curve.

Table 2. Results of the indirect treatment comparison for the PFS and OS hazard ratio of all possible MRCC treatments.

\begin{tabular}{|c|c|c|c|c|c|c|}
\hline Treatment & & Comparator & HR & BCI 95\% & $\begin{array}{c}\text { Number of } \\
\text { simulations }\end{array}$ \\
\hline \multicolumn{7}{|c|}{ Progression Free Survival (PFS) } \\
\hline SU & vS & IFN $\alpha$ & 0.543 & 0.45 & 0.648 & 5,000 \\
\hline BE/IFN $\alpha$ & vs & IFN $\alpha$ & 0.6761 & 0.60 & 0.758 & 5,000 \\
\hline PA & vs & IFN $\alpha$ & 0.572 & 0.45 & 0.717 & 5,000 \\
\hline
\end{tabular}




\begin{tabular}{|c|c|c|c|c|c|c|}
\hline BSC & vs & IFN $\alpha$ & 1.459 & 0.90 & 2.246 & 5,000 \\
\hline \multicolumn{7}{|c|}{ Overall Survival (OS) } \\
\hline SU & vs & IFN $\alpha$ & 0.8251 & 0.6758 & 1.009 & 5,000 \\
\hline BE/IFN $\alpha$ & vs & IFN $\alpha$ & 0.8605 & 0.5706 & 0.974 & 5,000 \\
\hline PA & vs & IFN $\alpha$ & 0.7525 & 0.6485 & 4.555 & 5,000 \\
\hline BSC & vs & IFN $\alpha$ & 1.953 & 0.6758 & 1.009 & 5,000 \\
\hline
\end{tabular}

Abbreviations: BE/IFN $\alpha$ : bevacizumab plus interferon alpha; BCI: Bayesian credibility interval; BSC: best supportive care; HR: hazard ratio, IFN $\alpha$ : interferon alpha; PA: pazopanib; SU: sunitinib; vs: versus.

\subsection{Costs}

For each health state, resources were identified, measured and valued within three main categories: (1) MRCC pharmacological treatment; (2) management of adverse events; and (3) disease monitoring/follow-up. It should be noted that adverse events were obtained from the pivotal trials of each possible comparator. Subsequently, to estimate the expected cost per event, a basket of resources was constructed considering all possible alternatives of management. It was assumed that in the presence of a grade 3 or 4 adverse events, the patient would consult to an emergency department. For the rest of the events (grade 1 and 2), it was assumed they were all managed on an outpatient basis and according to the indications during follow-up with the oncologist. The same number of follow-up visits were considered for each patient regardless the treatment, because the aim was to assess treatment response, disease progression and monitoring adverse effects. The baskets were built based on what was suggested in clinical guidelines and validated with oncologists according to the resources currently available in the Chilean public health system. The summary of costs per cycle are shown in Table 3.

Table 3. Expected cost per cycle (6 weeks) and category for each "First line" and "second line" treatment in 2015 US dollars

\begin{tabular}{|c|c|c|c|c|}
\hline \multirow{2}{*}{ Cost categories } & \multicolumn{3}{|c|}{ 1st line de treatment } & \multicolumn{2}{l|}{ Treatment after 1st line failure } \\
\cline { 2 - 5 } & Sunitinib & Pazopanib & Axitinib & BSC \\
\hline $\begin{array}{c}\text { Pharmacological } \\
\text { treatment }\end{array}$ & $\$ 2.973,80$ & $\$ 2.969,87$ & $\$ 7.926,75$ & \\
\hline
\end{tabular}




\begin{tabular}{|c|c|c|c|c|}
\hline Adverse Events & $\$ 165,96$ & $\$ 124,33$ & $\$ 61,55$ & \\
\hline Follow Up & $\$ 193,19$ & $\$ 193,19$ & $\$ 193,19$ & \\
\hline BSC management $^{1}$ & \multicolumn{3}{|l}{} & $\$ 434,26$ \\
\hline
\end{tabular}

Abbreviations: BSC: best supportive care.

NOTE: ${ }^{1}$ The average expected cost considers laboratory tests, mental health, medical procedures, hospitalization, images, medical supplies and medical visits with a specialist.

\subsection{Cost-effectiveness results}

The average total cost of treating a patient diagnosed with MRCC with SU is greater than treating the same patient with PA, which is higher than the cost of only providing BSC ( $\$ 45,786.29 ; \$ 43,255.05$; and $\$ 5,091.97$ respectively). It should be noted that minimal variations in drug prices produce important changes in the results which is also reflected in the small total incremental cost between SU and PA $(\$ 2,531.24)$. Similarly, the additional cost of treating a patient with PA instead of BSC is $\$ 38,163.08$, which is mainly explained by the price of the drug. It should be noted that less than $5 \%$ of the total cost involves the use of resources for the management of adverse events. The highest total cost associated with SU is explained by the fact that a smaller proportion of patients progress per cycle, thus increasing the period of treatment. This is reflected in an increase in the total expected cost of BSC in patients treated with PA.

A patient treated with SU accumulates in 10 years, on average, 1.399 QALYs and a patient treated with PA 1.37 QALYs leading to an incremental benefit of SU vs PA of only 0.03 QALY. The average ICER when comparing PA versus BSC is \$62,327.11/QALY and SU versus PA $\$ 85,885.22 /$ QALY (see Table 4). Both ICERs are on the efficiency frontier and above the suggested cost-effectiveness threshold in Chile of 1 GDP per capita (estimated at $\$ 18,346.66$ ).

The baseline scenario showed that the incremental health benefit is low when comparing PA versus SU (0.03 QALYs). Hence, it is expected that any variation of the price of the drug will significantly change the ICER. For this reason, the cost effectiveness results are presented 
varying the maximum price of SU and PA to $75 \%, 50 \%$ and $25 \%$ in relative terms (see Table 4). At all evaluated prices, the average ICER is above the threshold of 1 GDP per capita and at the lowest evaluated price (75\% reduction) the ICER of PA vs BSC and SU vs PA reaches $\$ 22,324.88$ and $\$ 40,664.05$ per additional QALY respectively.

Table 4. Cost effectiveness results: incremental analysis of sunitinib versus pazopanib and $\mathrm{BSC}$ as first line treatments for the base case and price reduction scenarios.

\begin{tabular}{|c|c|c|c|c|c|c|}
\hline Analysis & $\mathrm{TX}$ & Total cost & Total QALY & Inc Cost. & $\begin{array}{l}\text { QALY } \\
\text { Inc. }\end{array}$ & $\begin{array}{c}\text { ICER } \\
\text { (\$/QALY) }\end{array}$ \\
\hline \multirow{3}{*}{ Base Case } & BSC & $\$ 5,091.97$ & 0.757 & & & \\
\hline & PA & $\$ 43,255.05$ & 1.370 & $\$ 38,163.08$ & 0.61 & $\$ 62,327.11$ \\
\hline & SU & $\$ 45,786.29$ & 1.399 & $\$ 2,501.23$ & 0.03 & $\$ 85,885.22$ \\
\hline \multirow{3}{*}{$\begin{array}{l}25 \% \\
\text { reduction }\end{array}$} & BSC & $\$ 5,091.97$ & 0.757 & & & \\
\hline & PA & $\$ 35,090.56$ & 1.370 & $\$ 29,998.59$ & 0.61 & $\$ 48.993 .04$ \\
\hline & SU & $\$ 31,177.54$ & 1.399 & $\$ 2,086.98$ & 0.03 & $\$ 70,811.49$ \\
\hline \multirow{3}{*}{$\begin{array}{l}50 \% \\
\text { reduction }\end{array}$} & BSC & $\$ 5,091.97$ & 0.757 & & & \\
\hline & PA & $\$ 26,926.06$ & 1.370 & $\$ 21,834.09$ & 0.61 & $\$ 35,658.96$ \\
\hline & SU & $\$ 28,568.78$ & 1.399 & $\$ 1,642.72$ & 0.03 & $\$ 55,737.77$ \\
\hline \multirow{3}{*}{$\begin{array}{l}75 \% \\
\text { reduction }\end{array}$} & BSC & $\$ 5,091.97$ & 0.757 & & & \\
\hline & PA & $\$ 18,761.57$ & 1.370 & $\$ 13,669.60$ & 0.61 & $\$ 22,324.88$ \\
\hline & SU & $\$ 19,960.03$ & 1.399 & $\$ 1,198.46$ & 0.03 & $\$ 40,664.04$ \\
\hline
\end{tabular}

Abbreviations: BSC: best supportive care; ICER: incremental cost effectiveness ratio; Inc: incremental; PA: pazopanib, QALY: quality adjusted life years; SU: sunitinib; TX: treatment.

NOTE: treatment price reduction were relative to the published list price.

\subsubsection{Scenario Analysis}

The scenario analysis (Table 5) showed the impact on the results when $50 \%$ of the patients treated with SU and PA were treated with AX as second line upon progression. The estimated total expected cost of treating with SU and PA increased to $\$ 69,400.21$ and $\$ 67,936.89$, respectively and health benefits to 1.51 and 1.49 respectively. It should be noted that the incremental benefit of SU versus PA is lower compared to the baseline scenario (0.024 QALYs) 
leading to a higher ICER. The incremental analysis shows extended dominance of SU versus PA, thus resulting in an ICER of $\$ 85,446.97 /$ QALY when comparing SU versus BSC.

Table 5. Cost effectiveness results: scenario analysis assuming 50\% of patients' treated with SU/PA were given second line treatment.

\begin{tabular}{|c|c|c|c|c|c|}
\hline TX & Total cost & Total QALY & Inc Cost. & QALY Inc. & $\begin{array}{r}\text { ICER } \\
\text { (\$/QALY) }\end{array}$ \\
\hline BSC & $\$ 5,091.97$ & 0.757 & & 0.73 & $\$ 86,307.33$ \\
\hline PA & $\$ 67,936.89$ & 1.486 & $\$ 62,844.92$ & 0.02 & $\$ 59,831.92$ \\
\hline SU & $\$ 69,400.21$ & 1.510 & $\$ 1,463.32$ & & \\
\hline
\end{tabular}

Abbreviations: BSC: best supportive care; ICER: incremental cost effectiveness ratio; Inc: incremental; TX: treatment; PA: pazopanib; QALY: quality adjusted life years; SU: sunitinib.

\subsubsection{Deterministic sensitivity analysis}

The greatest variation of the ICER was observed when varying the OS HR based on the 95\% BCI of SU versus BSC reported from the MTC (95\% BCI 0.6485 to 4.555). The large amplitude of the interval reveals the great uncertainty associated with this parameter. When assuming the lowest value, the ICER was reduced to $\$ 34,518.97 / \mathrm{QALY}$ and when increased to the highest, the ICER reaches an estimated value of $\$ 213,676.82 / Q A L Y$. Other parameters that impacted the results were: the utility of SU while on treatment, the utility of BSC, the probability of receiving second line treatment, and the utility of SU while off treatment.

As the difference is so minimal in terms of total accrued QALYs when comparing SU versus PA, it was reasonable to assess a different scenario in which the utility of both interventions was varied. The results showed that a relative $10 \%$ reduction in the utility of PA, decreased the ICER of SU vs PA to $\$ 4,348.79 /$ QALY (incremental QALYs increased from 0.03 to 0.08 ). Similarly, if the utility of PA increases $10 \%$ in relative terms, the strategy became dominant. Hence, the ICER was very sensitive to minimal variations in the quality of life of one intervention versus another.

\subsubsection{Probabilistic sensitivity analysis}


A probabilistic sensitivity analysis was conducted using the four above mentioned prices. These were graphically represented in an incremental cost effectiveness plane (Figure 3-A and 3-B) where each point represents one simulation and the black and red line, the 1 and 3 GDP per capita threshold respectively. The set of ICERs distinguished in the plane come from the comparison between SU vs BSC and PA vs SU assuming the base case (Figure 3-A). Similarly, Figure 3-B shows the simulation results when the price of SU and PA was dropped $25 \%$.

Another way to represent the uncertainty of the results is through an acceptability curve (Figure 4-A and Figure 4-B). The probability of cost effectiveness at a 1 GDP per capita threshold was zero for the first three scenarios (baseline, $75 \%$ and $50 \%$ ). When the price of both strategies was reduced by $75 \%$, the probability of cost effectiveness reached approximately $40 \%$. This probability increased to more than $80 \%$ when the price of SU and PA was dropped at least 25\% considering a 3 GDP per capita threshold.

\section{Here Figure 3-A and 3-B}

\section{Here Figure 4-A and 4-B}

\section{Discussion}

The treatment and management of patients with MRCC until 2006 was very limited. However, in recent years, the development of new targeted drugs has shown to positively change the prognosis of these patients. Among them, SU and PA are the most recognized alternatives for the management of this disease. These alternatives offer patients more health in terms of years of life and quality of life, but have a very high associated cost that limits their use. The latter has motivated health systems to produce local evidence to answer the question whether or not is worth paying for this new but more expensive intervention. The objective of this study was to determine the cost-effectiveness of SU compared to PA and BSC from the 
perspective of the Chilean public health system in order to provide evidence to support decision making regarding the potential allocation of resources to finance this new therapeutic alternative.

SU and PA demonstrated important effects on PFS and quality of life of patients with MRCC. However, the results of this study showed that when comparing SU or PA vs BSC, considered the standard of care in Chile, the incremental cost of the new strategy was very high. This results in ICERs that are above the suggested cost-effectiveness threshold of 1 GDP per capita. On the other hand, when comparing SU vs PA, the expected incremental health benefit measured as QALYs, was very small favoring SU on average. However, the associated uncertainty around the utility estimates is large and can lead to opposite results where PA could be more effective than SU. From a clinical point of view, the evidence does not allow to affirm that one drug is more effective than the other, being the price is a determinant of costeffectiveness.

Our work extends to other economic evaluations conducted in different settings (mainly highincome countries) and assessing different treatment alternatives (SU, PA, IFN $\alpha, B E V / I F N \alpha$, TEM and SO) considering first and/or second line treatment of MRCC. Although most studies were cost effectiveness/utility, systematic reviews (4), cost minimization (1), cost analysis (1) and HTA related reports (3) were also found. Since PA became available, five costeffectiveness studies comparing it with SU have been also conducted for different settings[3437] with one utilizing real world data[38]. All those trial based studies concluded PA was costeffective compared to SU. In particular, one of these studies was later discussed by other authors who raised important limitations to the reported results that established PA as a dominant alternative compared to SU[39]. Their arguments were mainly focused on the utility estimates and sources of data used to conduct the survival analysis. In our study, we have further discussed these issues and the possible impact these limitations may have on study 
results. It should be noted that none of the available economic evaluations addressed the question relevant to health systems in lower income countries where the most likely available treatment is BSC. In fact, most Latin American countries do not have a reimbursement strategy in place for the use of SU or other MRCC targeted therapy, however many will consider exceptional mechanisms of access that lead to high expenditure and equity issues[40]. This is also consistent with the reality faced by some eastern European countries where, despite the incidence and mortality rates of kidney cancer are among the highest in the world, the access to first-line targeted therapies has been slow and has faced many obstacles[41].

This study has some limitations. The model uses utilities reported in the literature that were mainly derived from Anglo-Saxon population. It is well-known that valuation methods like time trade off reflect population's preferences that may not necessarily match with the Chilean population. Given the incremental health benefits (QALYs) obtained from this study are so small, the results are very sensitive to the source of utility used. However, given the lack of local utility data, values obtained from the British population were considered a reasonable alternative. In addition, the model assumption that treatment was immediately discontinued once progression was established, is also considered a limitation. In practice, this will depend on the time when the patient is assessed by the oncologist, which does not necessarily occur at the exact time of progression. This assumption may determine a bias that favors higher cost treatments like SU or PA.

The main source of uncertainty originates from the reported OS treatment effect from the study that compares PA versus PL[15-17]. The latter is partly explained by the cross-over of patients who initially received PL and upon progression, received PA, positively impacting the OS of patients. In fact, without adjustment, the HR did not show statistically significant differences (HR OS 0.91; 95\% CI 0.71-1.16: $\mathrm{p}=0.224$ ). To account for this structural 
uncertainty, the authors of this study provided two adjusted estimates obtained through two different methodologies, RPSFT and IPCW (inverse probability of censor weighting). However, both methods have advantages and disadvantages that increase the uncertainty in relation to this parameter. Despite this limitation, it is important to highlight that this parameter is useful and adequate from the perspective of an economic evaluation. The Bayesian statistical analysis, inherent to probabilistic sensitivity analysis, allows us to capture this uncertainty.

Cost-effectiveness analysis have been used by most, if not all, countries that currently have an HTA agency (or equivalent government body) to assess whether the use of first line treatment with targeted therapies, like SU or PA, represent good value for money. MRCC is an interesting case study as positive recommendations have been given despite high ICERs, based on other social values, mainly that MRCC is a relatively rare cancer with few available treatment options[43]. In the UK, SU was the first drug recommended after the adoption of a new guidance in 2009 for end-of life drug appraisal, which enables access to drugs for incurable illnesses. In this guidance, NICE supports the use of certain treatments with an ICER outside the threshold range of $£ 20,000$ - $£ 30,000 / Q A L Y$, if the patient population is small and there is a sufficient increase in survival[44]. This is a contentious discussion in countries that have an established HTA process to support decision-making, especially where judgments about distributive justice provided by cost-effectiveness have an important role in the decision itself. Unlike most previous studies, our analysis is more conservative and very cautious when concluding which alternative is more cost-effective. In fact, our results highlight important parameter uncertainties and limited evidence to recommend one alternative versus the other. Important is to emphasize that our study is the first to additionally compare SU and PA versus BSC, the standard of care in Chile and most developing countries, which was only possible after conducting an MTC. 


\section{Conclusion}

The results show that neither SU nor PA are cost-effective alternatives compared to the standard strategy or BSC from the perspective of the Chilean public health care system. However, in the case there is a willingness to finance any of these alternatives, for example, because they are considered orphan drugs, high cost or MRCC becomes a public health priority, then the question is which of the two (PA or SU) represents a better use of public resources. Due to the low incremental health benefit observed and the high second order uncertainty, it is reasonable to address this question as a cost minimization exercise, which is mainly determined by the price of each alternative.

\section{$6 \quad$ Key issues}

- SU nor PA are cost-effective alternatives compared to the standard strategy or BSC from the perspective of the Chilean public health care system.

- At a suggested threshold of 3 GDP per capita (approx. US\$55,041), the probability of costeffectiveness of SU, PA and BSC was 42\%, 39\% and 19\% respectively. This probability increases to $54 \%, 70 \%$ and an $82 \%$ when the SU price is reduced a $75 \%, 50 \%$ and $25 \%$ respectively.

- The decision between SU or PA is highly sensitive to the price of the drugs, rather than the outcomes.

- If there would be willingness to finance any of these alternatives, it is reasonable to address this issue as a cost minimization exercise, which is mainly determined by the price of each alternative.

\section{Funding}

This paper was funded by Pfizer Chile. This study was conducted under strict clauses of independence. The sponsors did not contribute at all in the development of this research. 


\section{Declaration of Interest}

C Vargas, M Espinoza and R Rojas disclose that as researchers they have conducted trainings and conferences on economic evaluations in health which have been financed by the pharmaceutical industry. As part of the academia I have also supported the Ministry of Health. The HTA unit as part of the university conducts studies financed by public and private sources including pharmaceutical companies. C Balmaceda discloses that the HTA unit as part of the university conducts studies financed by public and private sources including pharmaceutical companies. F Rodriguez discloses that they have supported the pharmaceutical chamber in Chile. I have previous experience working at the Ministry of Health in the elaboration of clinical guidelines. I have worked on several projects which were financed by the pharmaceutical industry, however, all of them have been conducted under strict clauses of independence. A Giglio has worked on projects which were financed by the pharmaceutical industry, however, all of them have been conducted under strict clauses of independence.

\section{Reviewer Disclosures}

Peer reviewers on this manuscript have no relevant financial or other relationships to disclose.

\section{Author contributions}

Study conception and design: Constanza Vargas, Manuel Espinoza

Acquisition of data: Carlos Balmaceda, Andres Giglio, Francisca Rodriguez, Ruben Rojas

Analysis and interpretation of data: Constanza Vargas, Manuel Espinoza, Carlos Balmaceda

Drafting of manuscript: Constanza Vargas, Manuel Espinoza

Critical revision: Constanza Vargas, Manuel Espinoza, Carlos Balmaceda, Francisca Rodríguez, Ruben Rojas

Final approval of the version to be published: Constanza Vargas, Manuel Espinoza, Carlos Balmaceda, Andres Giglio, Francisca Rodriguez, Ruben Rojas.

*All authors agree to be accountable for all aspects of the work.

\section{References}

Papers of special note have been highlighted as:

* of interest

** of considerable interest

1. Ljungberg B, Bensalah K, Canfield S et al. EAU guidelines on renal cell carcinoma: 2014 update. European urology, 67(5), 913-924 (2015).

2. Rini BI, Campbell SC, Escudier B. Renal cell carcinoma. The Lancet, 373(9669), 11191132 (2017). 
3. Who. Globocan 2012: Estimated cancer incidence, mortality and prevalence worldwide in 2012. (Ed.^ (Eds) (2012)

4. Pischon T, Lahmann PH, Boeing $\mathrm{H}$ et al. Body size and risk of renal cell carcinoma in the European Prospective Investigation into Cancer and Nutrition (EPIC). International journal of cancer, 118(3), 728-738 (2006).

5. Weikert S, Boeing $\mathrm{H}$, Pischon $\mathrm{T}$ et al. Blood pressure and risk of renal cell carcinoma in the European prospective investigation into cancer and nutrition. American journal of epidemiology, 167(4), 438-446 (2008).

6. Waalkes S, Merseburger AS, Kramer MW et al. Obesity is associated with improved survival in patients with organ-confined clear-cell kidney cancer. Cancer causes \& control : CCC, 21(11), 1905-1910 (2010).

7. Kovacs G, Akhtar M, Beckwith BJ et al. The Heidelberg classification of renal cell tumours. (Ed.^ (Eds) (England, 1997) 131-133.

8. Jonasch E, Gao J, Rathmell WK. Renal cell carcinoma. BMJ : British Medical Journal, 349 (2014).

9. Pérez Fentes DA, Blanco Parra M, Toucedo Caamaño V, Lema Grille J, Cimadevila García A, Villar Núñez M. Carcinoma renal metastásico de localización atípica: Revisión de la literatura. (Ed.^(Eds) (scieloes, 2005) 621-630.

10. Patel PH, Chaganti RSK, Motzer RJ. Targeted therapy for metastatic renal cell carcinoma. British Journal of Cancer, 94(5), 614-619 (2006).

11. Janowitz T, Welsh SJ, Zaki K, Mulders P, Eisen T. Adjuvant Therapy in Renal Cell Carcinoma-Past, Present, and Future(). Seminars in Oncology, 40(4), 482-491 (2013).

12. Fyfe G, Fisher RI, Rosenberg SA, Sznol M, Parkinson DR, Louie AC. Results of treatment of 255 patients with metastatic renal cell carcinoma who received high-dose recombinant interleukin-2 therapy. Journal of clinical oncology : official journal of the American Society of Clinical Oncology, 13(3), 688-696 (1995).

13. Motzer RJ, Hutson TE, Tomczak P et al. Sunitinib versus Interferon Alfa in Metastatic Renal-Cell Carcinoma. New England Journal of Medicine, 356(2), 115-124 (2007).*

14. Motzer RJ, Hutson TE, Tomczak $P$ et al. Overall survival and updated results for sunitinib compared with interferon alfa in patients with metastatic renal cell carcinoma. Journal of Clinical Oncology, 27(22), 3584-3590 (2009).**

15. Sternberg CN, Davis ID, Mardiak J et al. Pazopanib in locally advanced or metastatic renal cell carcinoma: results of a randomized phase III trial. Journal of clinical oncology : official journal of the American Society of Clinical Oncology, 28(6), 1061-1068 (2010).

16. Sternberg CN, Hawkins RE, Wagstaff J et al. A randomised, double-blind phase III study of pazopanib in patients with advanced and/or metastatic renal cell carcinoma: Final overall survival results and safety update. European Journal of Cancer, 49(6), 1287-1296 (2013).**

17. Motzer RJ, Hutson TE, Cella D et al. Pazopanib versus Sunitinib in Metastatic Renal-Cell Carcinoma. New England Journal of Medicine, 369(8), 722-731 (2013).**

18. Nice. Sunitinib for the first-line treatment of advanced and/or metastatic renal cell carcinoma. (Ed.^(Eds) (2009) 
19. Wu B, Dong B, Xu Y et al. Economic Evaluation of First-Line Treatments for Metastatic Renal Cell Carcinoma: A Cost-Effectiveness Analysis in A Health Resource-Limited Setting. PLoS ONE, 7(3), e32530-e32530 (2012).

20. Minsal. Guia metodologica para la evaluacion economica de ntervenciones en salud en Chile. (Ed.^(Eds) (2013)

21. Hudes G, Carducci M, Tomczak P et al. Temsirolimus, Interferon Alfa, or Both for Advanced Renal-Cell Carcinoma. New England Journal of Medicine, 356(22), 2271-2281 (2007).

22. Escudier B, Eisen T, Stadler WM et al. Sorafenib for treatment of renal cell carcinoma: Final efficacy and safety results of the phase III treatment approaches in renal cancer global evaluation trial. Journal of clinical oncology : official journal of the American Society of Clinical Oncology, 27(20), 3312-3318 (2009).

23. Collett D. Modelling Survival Data in Medical Research, Third Edition (2015).

24. Bcentral. Indicadores Diarios. (Ed.^ ${ }^{(E d s)}(2014)$

25. Minsal. Estudio verificación del costo esperado individual promedio por beneficiario del conjunto priorizado de problemas de salud con garantías explicitas 2012. (Ed.^(Eds) (Santiago, 2013)

26. Escudier B, Porta C, Schmidinger M et al. Renal cell carcinoma: ESMO Clinical Practice Guidelines for diagnosis, treatment and follow-up. Annals of oncology : official journal of the European Society for Medical Oncology, 25 Suppl 3, iii49-56 (2014).*

27. Cella D, Cappelleri JC, Bushmakin A et al. Quality of life predicts progression-free survival in patients with metastatic renal cell carcinoma treated with sunitinib versus interferon alfa. Journal of oncology practice, 5(2), 66-70 (2009).

28. Isp. Ficha de Producto: Sunitinib. (Ed.^(Eds) (2006)

29. Isp. Ficha de Producto: Pazopanib. (Ed.^ (Eds) (2013)

30. Motzer RJ, Escudier B, Tomczak P et al. Axitinib versus sorafenib as second-line treatment for advanced renal cell carcinoma: overall survival analysis and updated results from a randomised phase 3 trial. The Lancet. Oncology, 14(6), 552-562 (2013).

31. Jonsson L, Sandin R, Ekman M et al. Analyzing overall survival in randomized controlled trials with crossover and implications for economic evaluation. Value in health : the journal of the International Society for Pharmacoeconomics and Outcomes Research, 17(6), 707-713 (2014).

32. Ishak KJ, Proskorovsky I, Korytowsky B, Sandin R, Faivre S, Valle J. Methods for adjusting for bias due to crossover in oncology trials. PharmacoEconomics, 32(6), 533-546 (2014).

33. Amdahl J, Diaz J, Sharma A, Park J, Chandiwana D, Delea TE. Cost-effectiveness of pazopanib versus sunitinib for metastatic renal cell carcinoma in the United Kingdom. PloS one, 12(6), e0175920-e0175920 (2017).

34. Amdahl J, Diaz J, Park J, Nakhaipour HR, Delea TE. Cost-effectiveness of pazopanib compared with sunitinib in metastatic renal cell carcinoma in Canada. Current oncology (Toronto, Ont.), 23(4), e340-354 (2016). 
35. Capri S, Porta C, Delea TE. Cost-effectiveness of Pazopanib Versus Sunitinib as Firstline Treatment for Locally Advanced or Metastatic Renal Cell Carcinoma from an Italian National Health Service Perspective. Clinical therapeutics, 39(3), 567-580.e562 (2017).

36. Delea TE, Amdahl J, Diaz J, Nakhaipour HR, Hackshaw MD. Cost-Effectiveness of Pazopanib Versus Sunitinib for Renal Cancer in the United States. Journal of Managed Care \& Specialty Pharmacy, 21(1), 46-54 (2015).

37. Nazha S, Tanguay S, Kapoor A et al. Cost-utility of Sunitinib Versus Pazopanib in Metastatic Renal Cell Carcinoma in Canada using Real-world Evidence. Clinical drug investigation, 38(12), 1155-1165 (2018).

38. Benedict A, Ramaswamy K, Sandin R. Cost-Effectiveness of Pazopanib Versus Sunitinib for Renal Cancer in the United States. (Ed.^(Eds) (United States, 2015) 834-840.

39. Proyecto DIME. (Ed.^(Eds)

40. Vrdoljak E, Torday L, Szczylik C, Kharkevich G, Bavbek S, Sella A. Pharmacoeconomic and clinical implications of sequential therapy for metastatic renal cell carcinoma patients in Central and Eastern Europe. Expert Opin Pharmacother, 17(1), 93-104 (2016).

41. Chabot I, Rocchi A. How do cost-effectiveness analyses inform reimbursement decisions for oncology medicines in Canada? The example of sunitinib for first-line treatment of metastatic renal cell carcinoma. Value Health, 13(6), 837-845 (2010).

42. NICE. Appraising life-extending, end of life treatments. (Ed.^(Eds) (National institute for Health and Clinical Excellence).

\section{Relevant references}

13. Motzer RJ, Hutson TE, Tomczak $P$ et al. Sunitinib versus Interferon Alfa in Metastatic Renal-Cell Carcinoma. New England Journal of Medicine, 356(2), 115-124 (2007).*

Provides the treatment effect relevant to the intervention. Corresponds to the initial trial of sunitinib.

14. Motzer RJ, Hutson TE, Tomczak P et al. Overall survival and updated results for sunitinib compared with interferon alfa in patients with metastatic renal cell carcinoma. Journal of Clinical Oncology, 27(22), 3584-3590 (2009).**

Provides the updated treatment effect of sunitinib relevant to the intervention for overall survival.

16. Sternberg CN, Hawkins RE, Wagstaff J et al. A randomised, double-blind phase III study of pazopanib in patients with advanced and/or metastatic renal cell carcinoma: Final overall survival results and safety update. European Journal of Cancer, 49(6), 1287-1296 (2013).**

Provides the updated treatment effect of pazopanib relevant to the intervention for overall survival. It is the trial that allows the network provide treatment effect against BSC.

17. Motzer RJ, Hutson TE, Cella D et al. Pazopanib versus Sunitinib in Metastatic Renal-Cell Carcinoma. New England Journal of Medicine, 369(8), 722-731 (2013).**

Corresponds to the non-inferiority trial that compares sunitinib versus pazopanib. 
26. Escudier B, Porta C, Schmidinger M et al. Renal cell carcinoma: ESMO Clinical Practice Guidelines for diagnosis, treatment and follow-up. Annals of oncology : official journal of the European Society for Medical Oncology, 25 Suppl 3, iii49-56 (2014).*

Provides relevant current context of treatment alternatives. 


\section{Table and figure legends}

\section{Figure legends}

Figure 1. Time-dependent Markov model to represent the natural history of MRCC

Figure 1 represents the natural history of MRCC where patients transit through four possible health states: (1) patients receiving first-line treatment, (2) patients receiving second-line treatment, (3) patients receiving BSC and (4) specific-cause death.

1. Probability of not progressing and remaining in $1^{\text {st }}$ line treatment.

2. Probability of death given patient received $1^{\text {st }}$ line treatment.

3. Probability of disease progression

a. Probability of receiving $2^{\text {nd }}$ line given $1^{\text {st }}$ line treatment.

b. Probability of receiving BSC given 1 ${ }^{\text {st }}$ line treatment.

4. Probability of not progressing and remaining with second line treatment.

5. Probability of disease progression given patient received $2^{\text {nd }}$ line treatment.

6. Probability of death given BSC.

7. Probability of remaining in BSC.

Abbreviations: BSC: best supportive care; MRCC: metastatic renal cell carcinoma

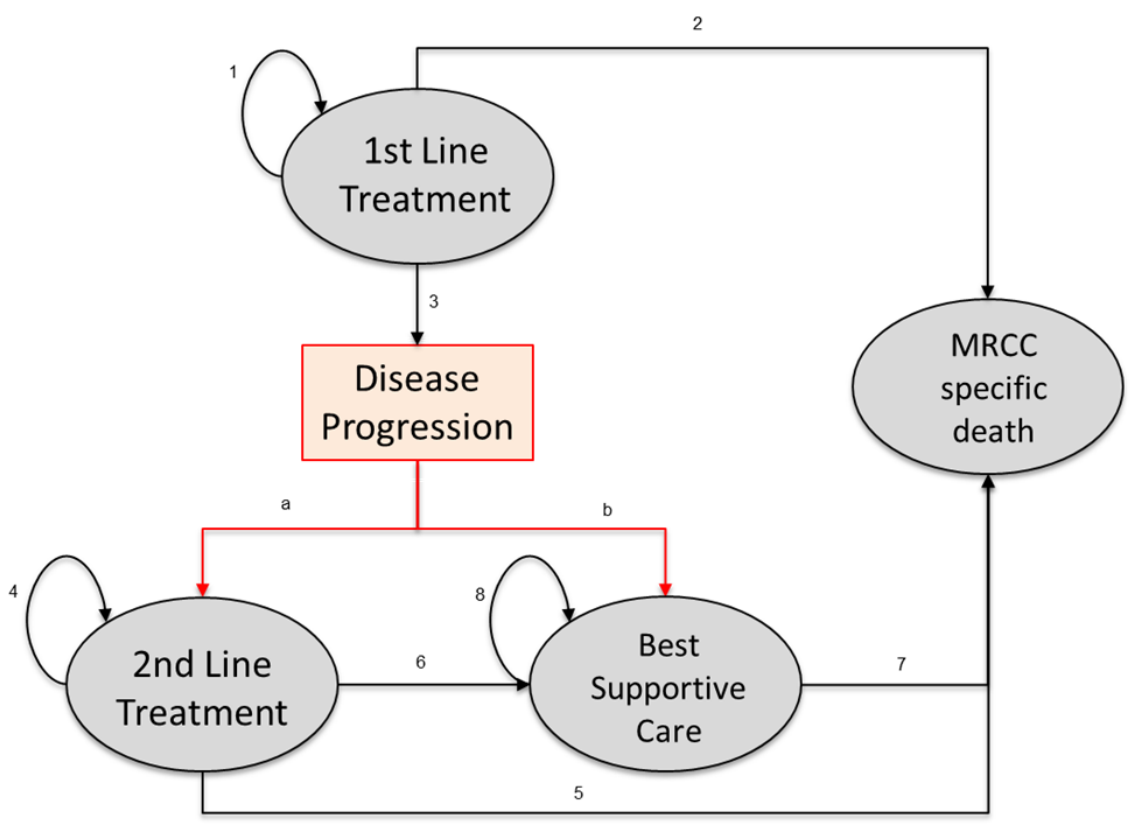

Figure 2A: Mixed treatment comparison network for PFS. 
Figure 2A represents the network for PFS consisting of five studies and five possible treatment alternatives for MRCC.

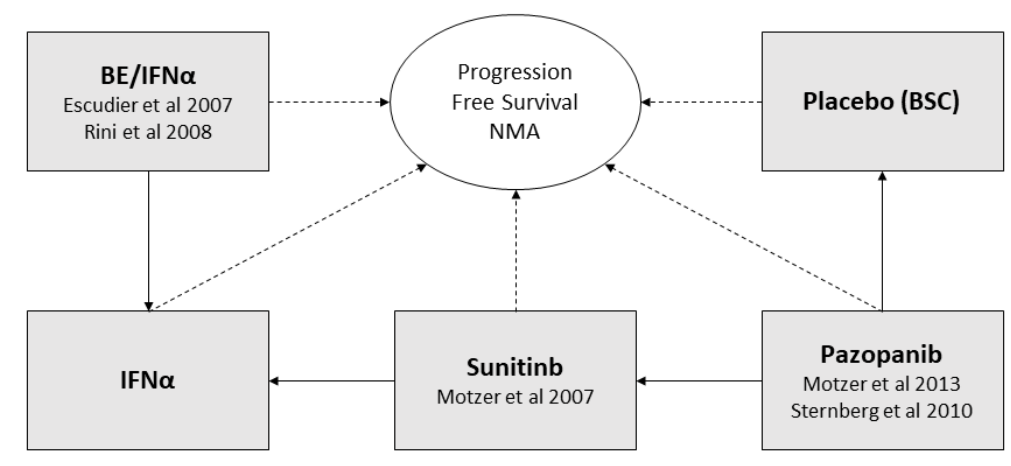

Abbreviations: BE: bevacizumab; IFN $\alpha$ : interferon alpha; BSC: best supportive care; MRCC: metastatic renal cell carcinoma; PFS: progression free survival.

NOTE: Dashed arrows represent indirect comparison and solid arrows represent direct compared evidence.

Figure 2B: Mixed treatment comparison network for OS.

Figure $2 \mathrm{~B}$ represents the network for OS consisting of five studies and five possible treatment alternatives for MRCC.

Abbreviations: BE: bevacizumab; IFN $\alpha$ : interferon alpha; BSC: best supportive care; MRCC: metastatic renal cell carcinoma; OS: overall survival.

NOTE: Dashed arrows represent indirect comparison and solid arrows represent direct compared evidence. 


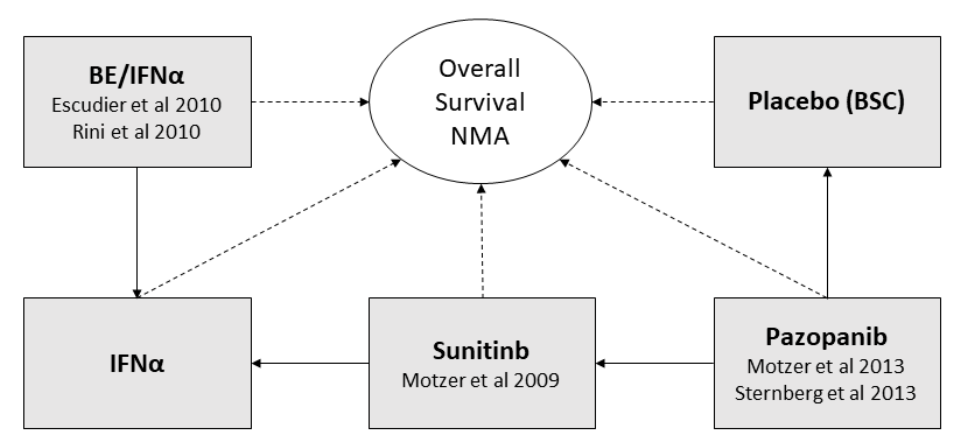

Figure 3-A and 3-B: Probabilistic sensitivity analysis: cost-effectiveness plane

Figure 3-A and 3-B represent the probabilistic sensitivity analysis for the base case and price reduction scenario for SU versus PA and PA versus BSC. Each dot represents 1 iteration of the model. Y-axis represents incremental costs. X-axis represents incremental QALYs. Diagonal lines represent the cost-effectiveness thresholds of 1 and 3 GDP per capita. Figure 3-B assumes the price of SU and PA were reduced $75 \%$ relative to the base case price.

Abbreviations: BSC: best supportive care; GDP, gross domestic product; ICER: incremental cost-effectiveness ratio; PA, pazopanib; QALYs, quality adjusted life years; USD, US dollars; SU, sunitinib; vs: versus. 

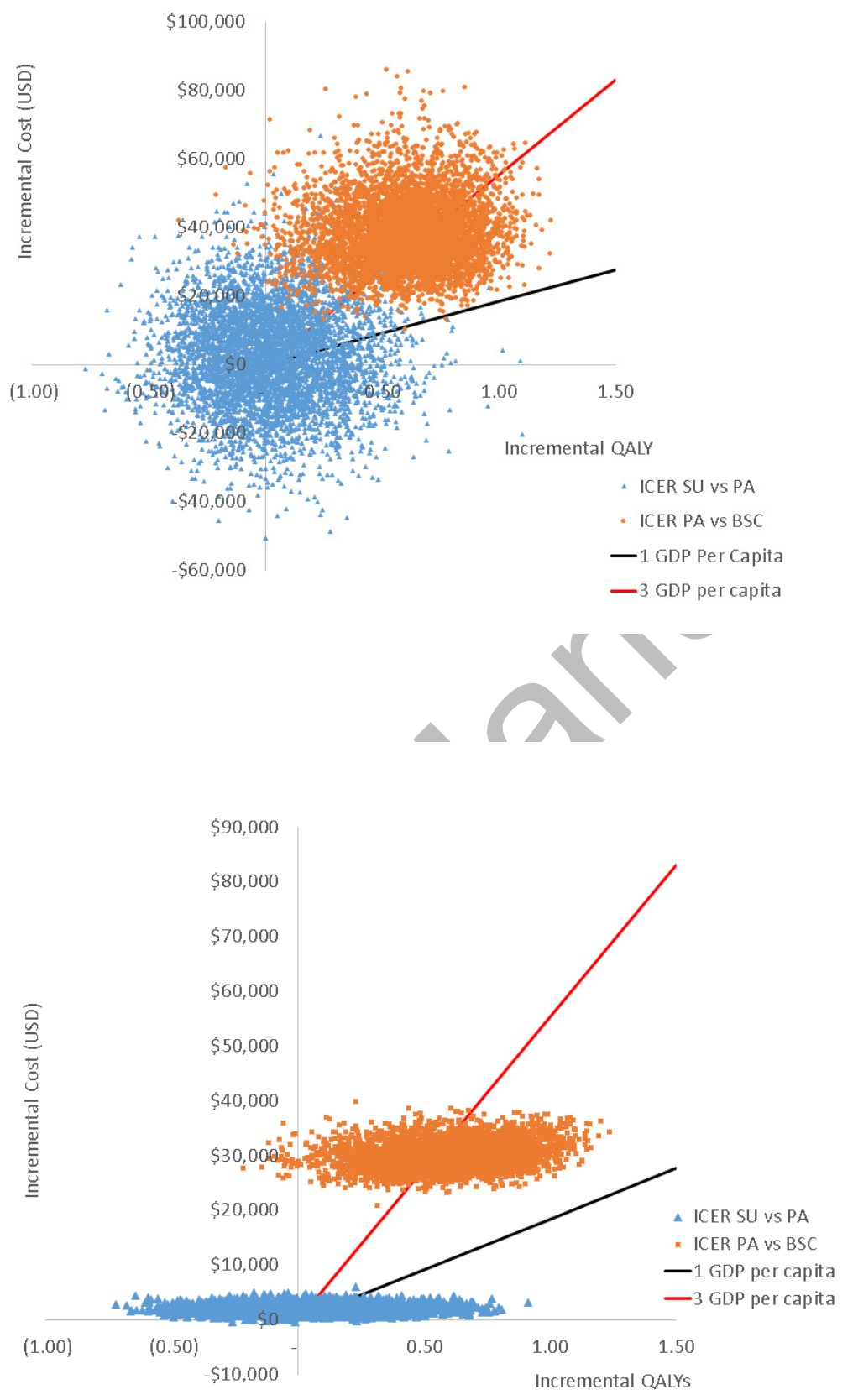

Figure 4-A and 4-B: Probabilistic sensitivity analysis: acceptability curve

Figure 3-A and 3-B represent the probabilistic sensitivity analysis for the base case and price reduction scenario for SU versus PA and PA versus BSC. Vertical lines represent the costeffectiveness thresholds of 1 and 3 GDP per capita. Y-axis represents the probability the 
intervention is cost-effective. $\mathrm{X}$-axis represents possible thresholds. Figure 3-B assumes the price of SU and PA were reduced $75 \%$ relative to the base case price.

Abbreviations: BSC: best supportive care; GDP, gross domestic product; PA, pazopanib; QALYs, quality adjusted life years; USD, US dollars; SU, sunitinib; vs: versus.
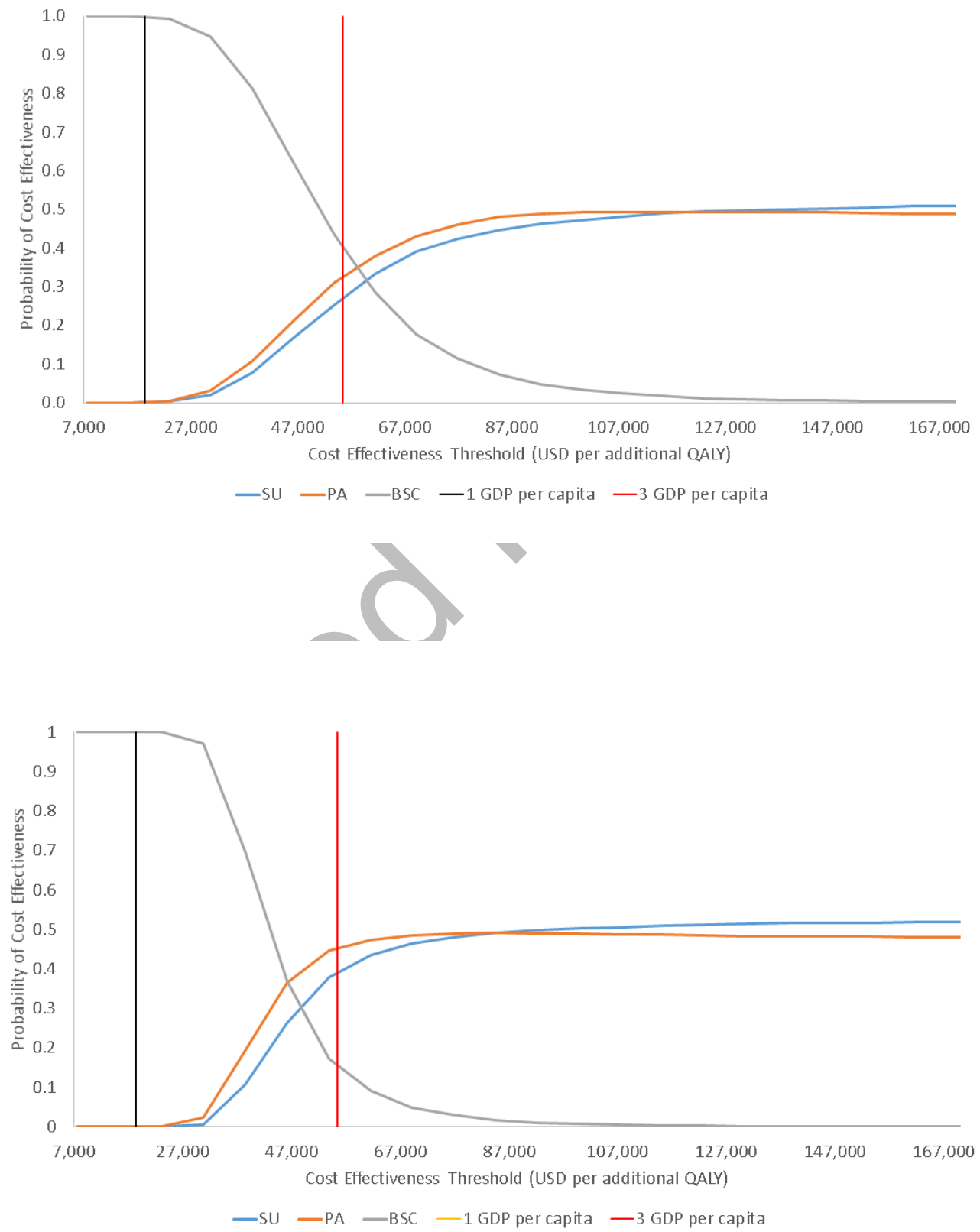


\section{Table legends}

\section{Table 1. Base line scenario: model input parameters and assumptions}

Abbreviations: BSC: best supportive care; HR: hazard ratio; IFN: interferon; IPD: individual patient data; mg; milligrams; N/A: not applicable; PA: pazopanib; PL: placebo; RPSFT: Rank Preserving Structural Failure Time; SU: sunitinib.

Table 2: Results of the indirect treatment comparison for the PFS and OS hazard ratio of all possible MRCC treatments.

Abbreviations: BE/IFN $\alpha$ : bevacizumab plus interferon alpha; BCI: Bayesian credibility interval; BSC: best supportive care; HR: hazard ratio, IFN $\alpha$ : interferon alpha; PA: pazopanib; SU: sunitinib; vs: versus.

Table 3: Expected cost per cycle (6 weeks) and category for each "First line" and "second line" treatment in 2015 US dollars

Abbreviations: BSC: best supportive care.

NOTE: ${ }^{1}$ The average expected cost considers laboratory tests, mental health, medical procedures, hospitalization, images, medical supplies and medical visits with a specialist.

Table 4: Cost effectiveness results: incremental analysis of sunitinib versus pazopanib and BSC as first line treatments for the base case and price reduction scenario.

Abbreviations: BSC: best supportive care; ICER: incremental cost effectiveness ratio; Inc: incremental; PA: pazopanib, QALY: quality adjusted life years; SU: sunitinib; TX: treatment.

NOTE: treatment price reduction were relative to the published list price.

Table 5: Cost effectiveness results: scenario analysis assuming $50 \%$ of patients were given second line treatment.

Abbreviations: BSC: best supportive care; ICER: incremental cost effectiveness ratio; Inc: incremental; TX: treatment; PA: pazopanib; QALY: quality adjusted life years; SU: sunitinib. 